\title{
Implementation of Augmented Reality as Information and Promotion Media on Dieng Tourism Area
}

\author{
Banu Nur Affan ${ }^{1, *}$, Agus Suryanto ${ }^{1}$, Arief Arfriandi ${ }^{1}$
}

\begin{abstract}
Information becomes necessary for tourists to get and improve the knowledge of tourism and their experiences about tourist destination. In Dieng tourism area, tourist information and promotional media has not been structured, as well as the need of media. The purpose of this research is produce Augmented Reality application that can visualize information of Dieng tourism, as interactive and interesting information, promotion tourism media. This research produces an android application which is used as information and promotion media of tourism in Dieng. Testing according to ISO 25010 with functional suitability got $100 \%$ suitability with Blackbox test, on portability aspect application successfully installed, update and uninstall on various smartphone environment, on performance efficiency aspect show average launch time is $1817 \mathrm{~ms}$ and also $0,22 \% \mathrm{CPU}$ and $177,8 \mathrm{MB}$ Memory usage. Results usability obtained SUS score 79.6 indicates the application get acceptable category to be used as information and tourism promotion media in Dieng.
\end{abstract}

Keywords - Augmented reality, Tourism, Promotion, ISO 25010 SUS

\section{INTRODUCTION}

$\mathbf{S}$ martphone users by the end of 2016 reach 2.10 Billion users or $28.7 \%$ of the world's total population, about $47.4 \%$ of mobile users will soon have smartphones by the end of this year, or about 2.32 Billion smartphone users from the entire population world [1]. With so many smartphone users in the world, many mobile phone companies concentrating in the production of smartphones compete to provide types and sorts of smartphones are innovative, futuristic and sophisticated in accordance with the needs and desires of consumers, especially with the advancement of the latest technology that can be adapted into the smartphone.

Since technology that can be adapted to smartphones is becoming more advanced, that opportunity can be used as an advantage to introduce a new technology [2]. One of the new technologies that are currently widely used in the development of creative and innovative media on smartphone devices is Augmented reality or AR technology. Augmented reality is one of expressive and creative new forms of media, where Augmented reality can enrich the way humans live their daily lives and extend human experience in the real world [3].

As an expressive and creative media, Augmented reality is worthy of use in various multidisciplinary areas such as simulation, education, entertainment, health, and games [4]. In addition, $\mathrm{AR}$ is being developed as part of a smart tourism information media to provide information on destinations and

${ }^{1}$ Department of Electrical Engineering, Faculty of Engineering, Semarang State University, Sekaran, Gunungpati, Semarang, Indonesia.

Correspondence to T. Author, email: banu.affan@yahoo.com. Tel.:+6282242477184. attractions or events, and demonstrate its potential to become a new tourism service [5]. A research conducted by Marimon at 2010 in a journal entitled MobiAR: Tourist Experience Through Mobile Augmented reality states, tourists tend to see mobile apps as well as gadgets that have information about locations, activities and historic places [6].

Tourism field continues to grow as well as with the number of tourists who seek information will be the location of the tour that will be visited, Augmented reality technology can be used as a medium conveyance of information about the elements of tourist locations in question. With $\mathrm{AR}$ technology, help tourist attractions to be visited more familiar when other tourists go to these attractions [2]. In addition to the use of Augmented reality as a medium of information, Augmented reality can also be used as a media campaign with innovative, creative and interactive form to make users of applications entertained and accepted smartphone users as well [7]. The form of interactive promotional media and Augmented reality advertising, allows users to properly engage with advertising, so everything becomes private and directly creates connections with users [8].

Therefore, an idea arises to implement an AR application as information media and promotion of tourism, in the hope that it can be used as a tool for tourists who are traveling to explore and add deeper knowledge about the tourist information being visited, as well as promoting tourism as well as events especially in the tourist area of Dieng.

Dieng tourism area is chosen because it has a tourist sites consisting of various types ranging from natural attractions, historic heritage and so on, many tourists from local and foreign, and less optimal use of technology as a creative and innovative media in the delivery of information about the tourist attraction as well as tourist events in the tourist area of Dieng where so far rely on conventional media such as brochures and social media. With so expected Augmented reality applications as a media of information and tourism promotion in the tourist area of Dieng, can help solve existing problems.

\section{METHODS}

Research method used in this research is Research and Development (R \& D). Research and Development research method is a research method used to produce a specific product and test the effectiveness or feasibility of the product [9].

Research and Development method in this research aim to develop an application of Augmented reality as media of information and promotion. This research method is done with several stages: potential and problem, literature study, product development, expert validation, product revision, product trial, product revision, user response, product revision 
and final product.

Flow diagram of research process with research and development method can be seen in Figure 1.

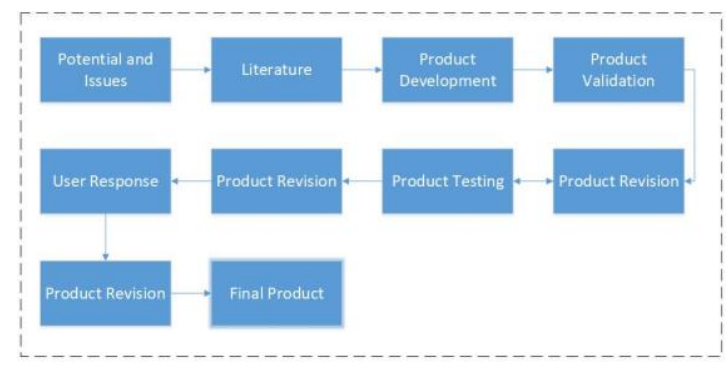

Fig. 1. Steps of Research Methods

In the product development stage, research uses the Waterfall development model. Waterfall or so-called Linear Sequential Model is a model developed in the development of a software or application. In the Waterfall model, the development stage is systematically from one stage to another such as the waterfall model.

This development model proposes an approach to systematic and sequential software development, from needs analysis, design, coding, testing and maintenance. This development model is linear from the early stages of software development is the stage of planning until the final stage of system development is the maintenance phase. Support or maintenance stage is where the application or software already in the hands of customers.

Figure 2 is a step diagram of the application development process or software with the Waterfall model.

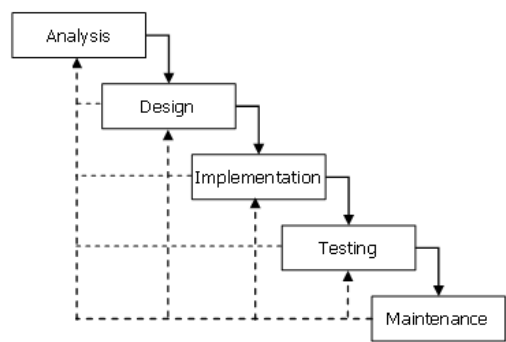

Fig. 2. Steps of Application Development Dieng AR

\section{RESULTS}

\section{A. Results of Software Development}

After passing the stage of analysis, design, implementation of code, testing and maintenance, then got the end result of the development of a product application Dieng AR. When you first open Dieng AR application, it will appear splash screen and loading screen shown in Figure 3.

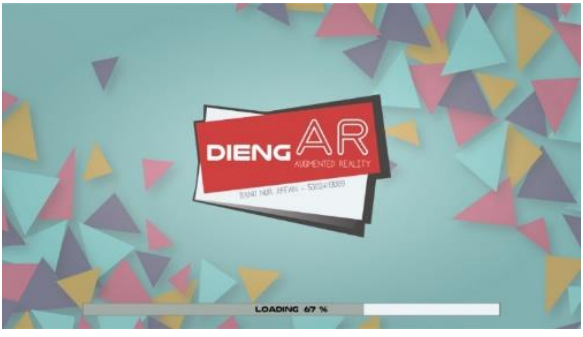

Fig. 3. Loading Dieng Screen AR

After loading process, the page appears select the language of the application as in Figure 4, this serves as the language settings of the application interface, when choosing the Indonesian language, then the language used from the start menu till the AR page with Indonesian, and vice versa.

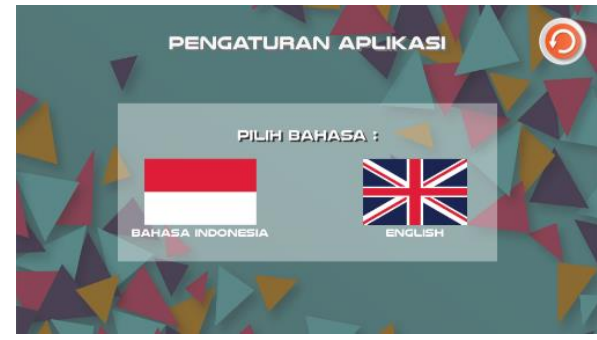

Fig. 4. Dieng AR language preference

The main menu of Dieng AR application there are various buttons used to go to AR page, tour menu, download Marker, help, about, until setting button and exit application as in picture 5. Display language used in main menu according to language selection.

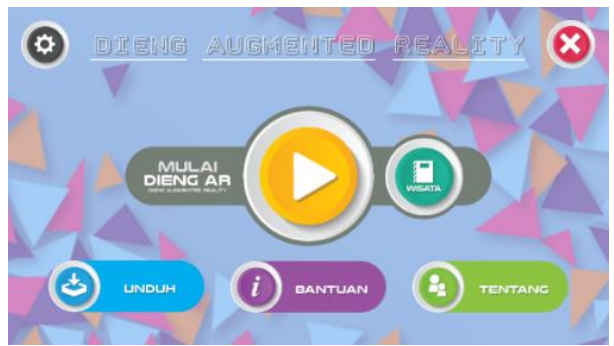

Fig. 5. Dieng AR main menu

In Augmented Reality technology, especially the most important Marker-based Augmented Reality is its marker as a virtual object augmentation reference. Dieng AR using the natural Marker located in the tourist area of Dieng, for that should visit to Dieng, or in the application there is a Marker download menu like figure 6 which provides a link to download images or markers that used, if user not found the images or markers that application used in the region Dieng tours sites. 


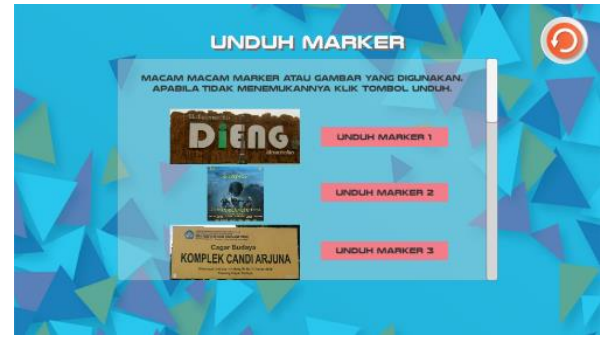

Fig. 6. Markers used Dieng AR

Marker when scanned with Dieng AR application, which was only object marker and ordinary paper, when scanned with Dieng AR application, will appear information about the tourist object or tourist event out automatically above the markers (figure 7), on this information display is also available button to play Video and view other information. Display language information that appears according to language selection.

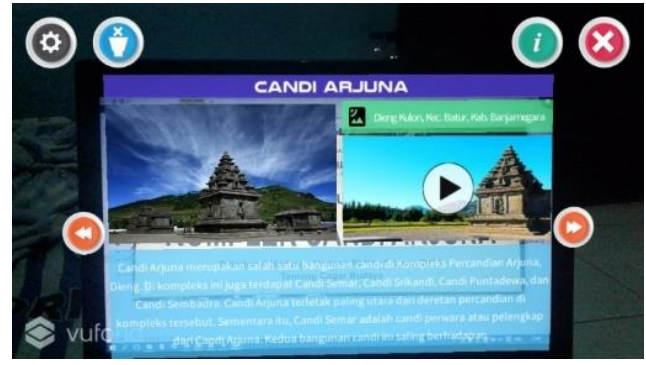

Fig. 7. Information appears during augmentation

Dieng AR application uses a variety of markers which are taken from the original image in the Dieng tourist attraction. The process of scanning Marker with Dieng AR application is figured out at figure 8 which is done outdoors.

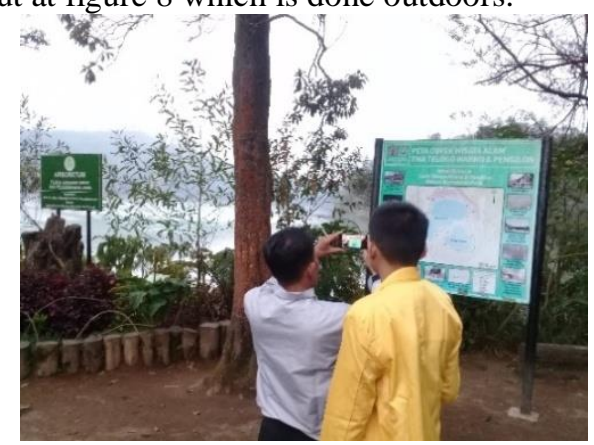

Fig. 8. Scanning a natural Marker in Dieng

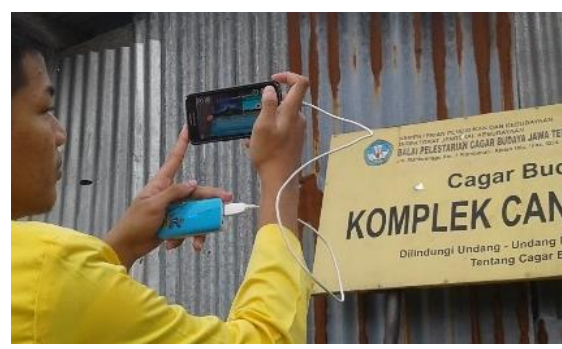

Fig. 9. Dieng AR display when scanning outdoor

Similar to the usual Marker scans, Dieng AR applications when outdoor scanning runs well and detected as well too, with the display of information content tailored to the Marker detected on each tourist sites, figure 9.

\section{B. Testing}

Software quality can be assessed through specific method measures and methods, as well as through software testing. One of the software quality measures is ISO 25010, created by the International Organization for Standardization (ISO) and the International Electrotechnical Commission (IEC). ISO 25010 replaces the previous standard of ISO 9126 [10], altered some classifications while still maintaining generalized hierarchical parsing [11].

ISO 25010 establishes eight characteristics of functional suitability, reliability, performance efficiency, usability, maintainability, security, compability and portability which are subdivided into a series of sub-characteristics [10].

This study uses four characteristics that exist in ISO 25010 , there are functional suitability, portability, performance efficiency and usability. The selection of these 4 characteristics is in accordance with the test of the device consisting of various aspects of test presented by Assaf Ben David, there are functional testing, compatibility testing (definition of compatibility that David said has a common understanding with aspects of Portability ISO 25010), Usability testing, performance testing, security testing, operability testing [14], as well as Segue Technologies Quality Control team, which revealed that the category in performing mobile applications consists of functional tests, compatibility tests (meaning compatibility presented by Seageu Tech has similar understanding with aspects of Portability ISO 25010), performance tests and Usability tests [15].

1) Functionality Suitability testing result : Tests on aspects of Functionality Suitability, using Blackbox test and validation by experts.

In the Blackbox test where test results are obtained with scenarios of 34 test scenarios, yielding $100 \%$ of successful or valid test scenarios, all scenarios correspond to expected results.

Expert validation in knowing the validity of this application using a questionnaire. Questionnaires were given to experts in the field of software engineering and stakeholders who knew about the tourist area of Dieng, selected to test the application. Table 1 shows the percentage of application validation by media and material experts.

Table 1. Table heading here.

\begin{tabular}{llcl}
\hline \hline No. & Validation Experts & Percentage & Criteria \\
\hline 1. & Media Experts & $90,27 \%$ & very well \\
2. & Content Experts & $82,95 \%$ & very well \\
\hline & Average & $86,61 \%$ & very well \\
\hline \hline
\end{tabular}

The average score of $86.61 \%$ validation results, which means the media meet the criteria very well / deserve to be used as a medium of information and promotion and testing to the next stage. 
2) Portability testing result: Test portability there are 3 sub-characteristics according to ISO 25010, namely adaptability, installability and replacability tested.

Portability testing on Dieng AR application is divided into 3 stages,

1. Installs and runs in different devices with different OS.

2. Installs and runs in various devices with different screen sizes.

3. Install and run in various devices and then updated with new versions of the application

Testing portability number 1 and 2 using Cloud Testing Google Firebase Test Labs consisting of 35 smartphone devices with various versions of android systems, smartphone brands, screen sizes and other specifications. While testing installs, runs and updates on various physical devices or directly, with 5 different smartphone devices.

1. Based on the results of portability testing by installing and running smartphone devices with different versions of OS from Cloud Testing Google Firebase Test Labs obtained a percentage of success of 100\% shown in Figure 10.

Portability of various versions of the Android OS

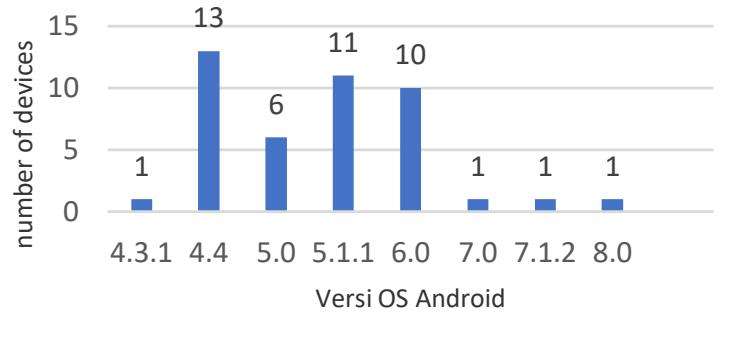

Fig. 10. Dieng AR portability test chart of various Android OS

2. Based on the results of portability testing by installing and running smartphone devices of various screen sizes from Cloud Testing Google Firebase Test Labs obtained a percentage of success of 100\% shown in Figure 11.

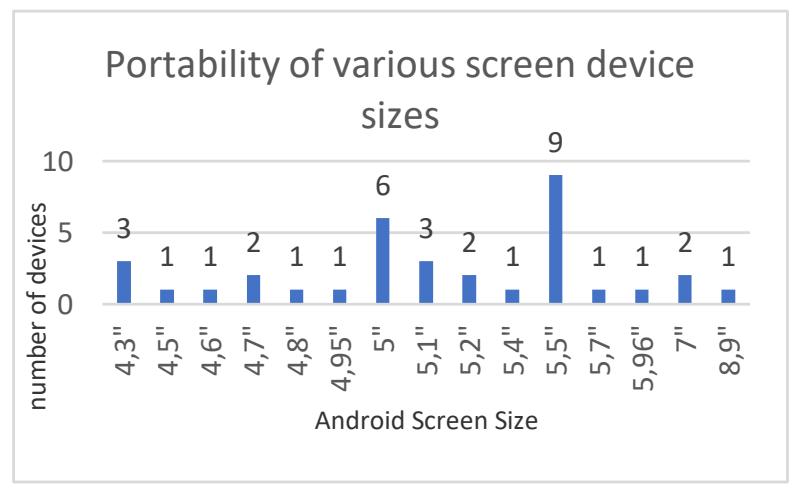

Fig. 11. Dieng AR portability test charts of various screen device sizes

3. Portability testing results by installing, running and updating smartphone devices of various brands, OS version and screen size of 5 devices obtained a percentage of success of $100 \%$ shown in table 2 .
Tabel 2. a percentage of success of $100 \%$

\begin{tabular}{|c|c|c|c|c|c|c|}
\hline No. & Smartphone & OS & $\begin{array}{l}\text { Screen } \\
\text { Size }\end{array}$ & Install & Run & Update \\
\hline 1. & Samsung J2 & 5.1 .1 & $4,7 ”$ & $\sqrt{ }$ & $\sqrt{ }$ & $\sqrt{ }$ \\
\hline 2. & Oppo A37f & 5.1 .1 & $5 "$ & $\sqrt{ }$ & $\sqrt{ }$ & $\sqrt{ }$ \\
\hline 3. & $\begin{array}{l}\text { Lenovo } \\
\text { A6000 }\end{array}$ & 4.4.4 & $5 "$ & $\sqrt{ }$ & $\sqrt{ }$ & $\sqrt{ }$ \\
\hline 4. & $\begin{array}{l}\text { Xiaomi } \\
\text { Redmi 3S }\end{array}$ & 6.0 .1 & $5 "$ & $\sqrt{ }$ & $\sqrt{ }$ & $\sqrt{ }$ \\
\hline 5. & $\begin{array}{l}\text { Sony Xperia } \\
\text { ZR }\end{array}$ & 5.1 .1 & $4,5 ”$ & $\sqrt{ }$ & $\sqrt{ }$ & $\sqrt{ }$ \\
\hline
\end{tabular}

Based on the overall portability test results, it can be concluded that Dieng AR applications can be installed, run, updated and un-installed on different device environments.

3) Performance Efficiency testing result : The Performance Efficiency testing are tested 2 subcharacteristics according to ISO 25010, ie time behavior and resource usage.

Performance testing is divided into 2 stages, there is

1. Calculates application launch time when first started.

2. Calculates smartphone resource usage when the app is running.

Performance test number 1 and 2 tested manually using 5 smartphone devices with different specifications. Then with stopwatch and system monitor app helps, calculates the application performance starting from launch time and resource usage. Here are the results of both performance experiments performed,

1. From the performance test in the sub category of time behavior, the average time of launch time required for applications on the 5 different devices is 1817 milisecond shown in Figure 12.

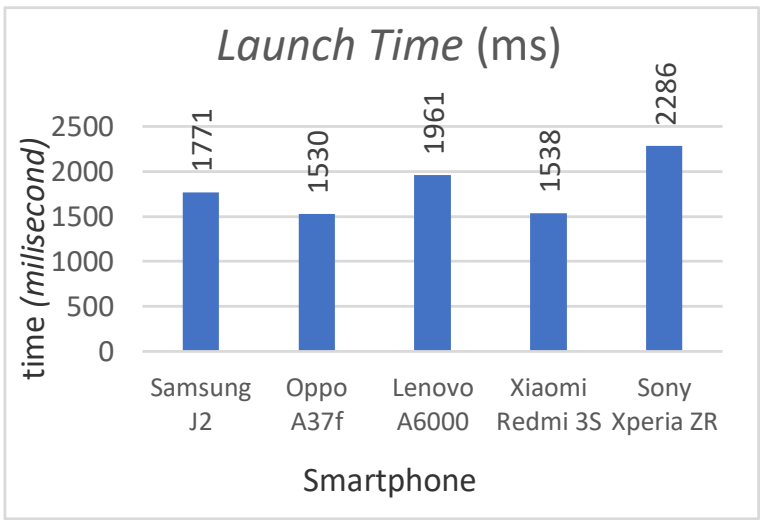

Fig. 12. Dieng AR performance launch test graphs from various devices

2. From the performance efficiency test in the usage resource sub-category, the average use of CPU and application memory on 5 different devices when running Dieng AR application is $0,22 \%$ CPU usage and $177,8 \mathrm{MB}$ memory usage is shown in Figures 13 and 14. 


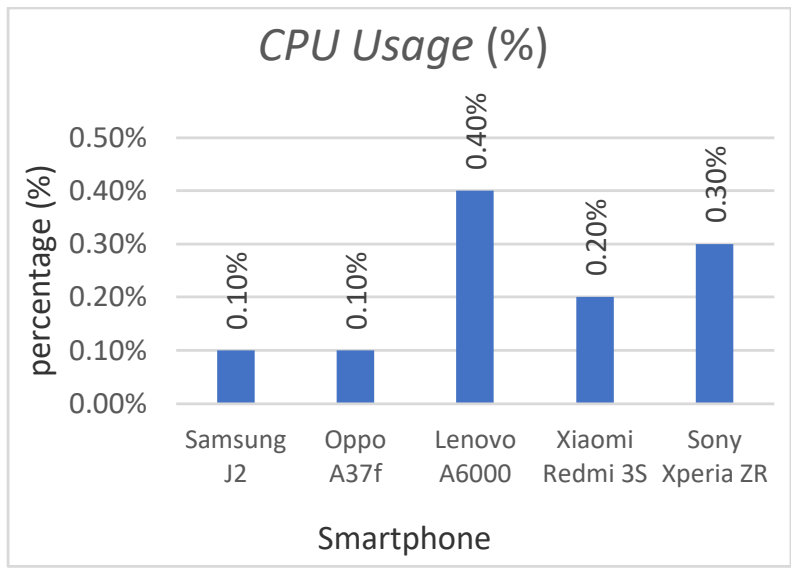

Fig. 13. Dieng AR CPU usage performance graph of various devices

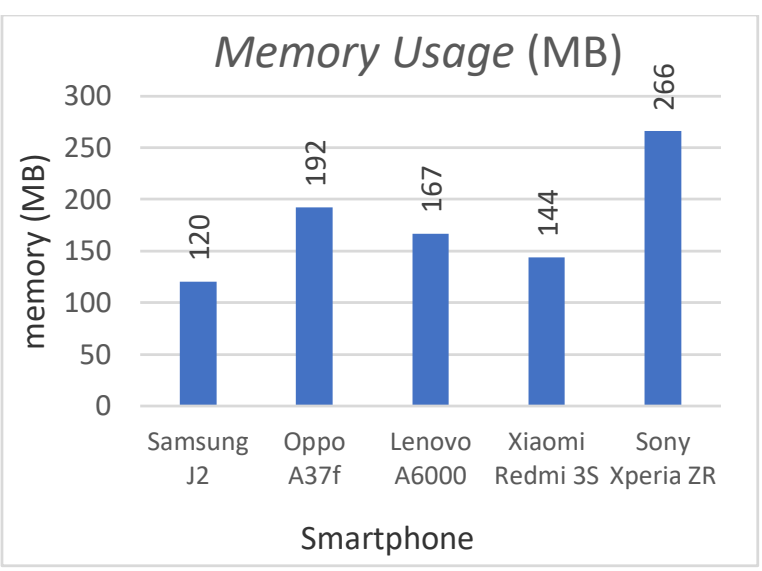

Fig. 13. Performance test graph of Dieng AR Memory usage from various devices

Application performance test results show launch time with an average launch time of 1817 milisecond with the fastest launch time of 1530 milisecond and slowest launch 2286 milisecond and resource use with an average of $0,22 \%$ use CPU and $177,8 \mathrm{MB}$ memory usage, with $0,1 \%$ lowest CPU usage resource and $0,4 \%$ at the most CPU usage, while the lowest memory usage is $120 \mathrm{MB}$ and $269 \mathrm{MB}$ highest when the tested device run Dieng AR application.

4) Usabiliy testing result : Usability testing in this study using user responses implemented in the area of tourist areas Dieng, by involving tourists, as well as the general public, with many users a number of 50 users.

User response in this study using usability testing, where to know the user response Dieng AR application as a media of information and tourism promotion in the Dieng tourist sites.

The results of user responses are carried out with calculations according to the guidelines of the SUS (System Usability Scale) processing [12]. The calculation of SUS score results get the final average score of 79.6, where the score goes in the score range $>70$ which means, Dieng AR application entered in the criteria acceptable or feasible to use [13].

Based on the results of research, Augmented reality application as a medium of information and promotion in the tourism area of Dieng is feasible and easy to use and become an interesting and interactive application for users both tourists and the general public in digging information about tourist objects and tourist events, this is supported by validation experts and results of satisfactory suitability, portability and performance functionality tests show good results and user-accepted reusability and user response, as well as research supported by Lashkari [2], which states that Augmented reality technology can be an application that can be easily used and can make people interested about what is promoted.

The use of augmented reality technology in the field of tourism becomes an innovative and interesting innovation both as a medium of information for tourists and as a promotional tool for tourist areas and tourist attractions, especially with supported applications that can visualize information with about the tour is well, interesting and interactive.

\section{CONCLUSION}

Research by developing this Dieng Augmented reality application, using Vuforia SDK and Unity framework. This app requires a natural Marker that has been specified as an augmentation reference. Application design phase using Flowchart and UML for software design that includes usecase diagram and Activity Diagram.

Dieng AR applications are tested using ISO 25010 quality standards and validated by media and material experts. On the functional aspect it gets $100 \%$ percentage. In the validation by media experts and materials get a percentage of $86.61 \%$ with very good category. Testing portability, the application works $100 \%$ for use in various device environments. The application performance test gets the average launch time of $1817 \mathrm{~ms}$ and the average is $0,22 \% \mathrm{CPU}$ usage and $177,8 \mathrm{MB}$ memory usage. In the user response stage, get the SUS value of 79.6 with acceptable category or eligible to be used as a media of information and tourism promotion in Dieng area.

\section{ACKNOWLEDGMENT}

The authors acknowledge the Government Tourism Office of Wonosobo District, for their support on this research.

\section{REFERENCES}

[1] Mahajan, A. C. Internet Users Worldwide 2016 - 2020: The Growth At Decreasing Rate [REPORT]. [Online] Available at: https://dazeinfo.com/2016/06/13/number-internet-users-worldwide2016-2020/. 2016

[2] Lashkari, A. H., Parhizkar, B. \& Mohamedali, M. A. Augmented Reality Tourist Catalogue Using mobile technology. Kuala Lumpur, Second International Conference on Computer Research and Development, Kuala Lumpur. 2010.

[3] Becker, G. Challenge, Drama \& Social Engagement: Designing, San Fransisco: Web 2.0 Expo. 2010.

[4] Noh, Z., Sunar, M. S. \& Pan, Z. A Review on Augmented Reality for Virtual Heritage System. In: International Conference on Technologies for E-Learning and Digital Entertainment. Berlin: Springer. 2009.

[5] Chung, N., Han, H. \& Joun, Y. Tourists intention to visit destination: Role of augmented reality applications for heritage site. Computers in Human Behavior. 2015.

[6] Marimon, D. MobiAR: Tourist Experiences through Mobile Augmented Reality, Barcelona, Spain: Telefonica Research and Development. 2010. 
[7] Adha, Y. A. Model Penerimaan Teknologi Augmented Reality Sebagai Media Komunikasi Pemasaran (Studi Kasus Penerimaan iButterfly Oleh Pengguna Smartphone). Depok: UI. 2012.

[8] Corral, R. The Benefits of Augmented Reality and Interactive Advertising. [Online] Available at: www.quickmarketing.com/thebenefits-of-augmented-reality-and-interactive-advertising/[Accessed 31 May 2017]. 2015.

[9] Sugiyono. Metode Penelitian Dan Pengembangan (Research And Development / R\&D). Bandung: Alfabeta. 2015.

[10] ISO/IEC-25010. iso25000.com. [Online] Available http://iso25000.com/index.php/en/iso-25000-standards/iso-25010 [Accessed 20 July 2017]. 2011.

[11]Wagner, S., 2013. Software Product Quality Control. New York: Springer Heidelberg.

[12]Lewis, J. R. \& Sauro, J. The Factor Structure of the System Usability Scale. International conference on human centered design. 2009: pp. 94 103.

[13] Bangor, A., Kortum, P. T. \& Miller, J. T. An Empirical Evaluation of the System Usability Scale. International Journal of Human-Computer Interaction. 2008; 6(24): 574-594.

[14]David, A. B. Mobile App Testing. In: J. Diaz, ed. Testing Experience. Berlin: Diaz Hiltersheid. 2011; 64-65.

[15]Team, T. S. Q. C. Testing Mobile Apps for Functionality and Usability. [Online] Available at: http://www.seguetech.com/testing-mobile-appsfunctionality-usability/[Accessed 3 Agustus 2017]. 2016. 